\title{
Secondary CDUS Contact
}

National Cancer Institute

\section{Source}

National Cancer Institute. Secondary CDUS Contact. NCI Thesaurus. Code C51870.

A subordinate person within the Cooperative Group, institution, clinical site, or/and

particular study that is responsible for the clinical trial data completion and submission to $\mathrm{NCl}$ via Clinical Data Update System (CDUS). 